\title{
Uniqueness and nonuniqueness for the approximation of quasilinear elliptic equations
}

\author{
André, $\mathrm{N}$; Chipot, $\mathrm{M}$
}

\begin{abstract}
We investigate the issue of uniqueness and nonuniqueness for the approximate solution of quasilinear elliptic equations. In particular we show that even if the continuous problem admits a unique solution, its approximation by finite elements may lead to several approximate solutions. @1996 Society for Industrial and Applied Mathematics
\end{abstract}

DOI: https://doi.org/10.1137/S0036142994267400

Posted at the Zurich Open Repository and Archive, University of Zurich ZORA URL: https://doi.org/10.5167/uzh-22529

Journal Article

Originally published at:

André, N; Chipot, M (1996). Uniqueness and nonuniqueness for the approximation of quasilinear elliptic equations. SIAM Journal on Numerical Analysis, 33(5):1981-1994.

DOI: https://doi.org/10.1137/S0036142994267400 


\title{
UNIQUENESS AND NONUNIQUENESS FOR THE APPROXIMATION OF QUASILINEAR ELLIPTIC EQUATIONS*
}

\author{
N. ANDRÉ ${ }^{\dagger}$ AND M. CHIPOT ${ }^{\ddagger}$
}

\begin{abstract}
We investigate the issue of uniqueness and nonuniqueness for the approximate solution of quasilinear elliptic equations. In particular we show that even if the continuous problem admits a unique solution, its approximation by finite elements may lead to several approximate solutions.
\end{abstract}

Key words. approximation, quasilinear elliptic equations, finite elements

AMS subject classifications. 35JXX, 65N30

1. Introduction. Let $\Omega$ be a polygonal bounded open subset of $\mathbf{R}^{n}, n \geq 1$. Assume that $a(x, u)$ is a Carathéodory function satisfying

$$
0<\alpha \leq a(x, u) \leq \beta \text { almost everywhere (a.e.) } x \in \Omega \forall u \in \mathbf{R},
$$

where $\alpha, \beta$ are two positive constants. For $f \in H^{-1}(\Omega)$ consider the problem

$$
\left\{\begin{array}{l}
-\frac{\partial}{\partial x_{i}}\left(a(x, u) \frac{\partial u}{\partial x_{i}}\right)=f \text { in } \Omega, \\
u \in H_{0}^{1}(\Omega) .
\end{array}\right.
$$

We use the summation convention and we refer to [GT] or [KS] for the definition of the Sobolev spaces used throughout the paper.

First, under the above assumptions, using a fixed-point argument of Schauder type, it is very easy to show that (1.2) admits a solution (see for instance [CM]). Moreover, we have the following theorem.

THEOREM 1.1. Assume that for some positive constant $C$ one has

$$
|a(x, u)-a(x, v)| \leq C|u-v| \quad \forall u, v \in \mathbf{R} \text {, a.e. } x \in \Omega .
$$

Then the problem (1.2) has a unique solution.

We refer to $[\mathrm{AC}],[\mathrm{T}]$, or $[\mathrm{GT}]$ for a proof. Note that some extensions of this theorem in terms of the modulus of continuity of $a$ are possible (see [CM], [AC], [Ar], [BKS], [CC], $[\mathrm{M}]$ ) and in the case where (1.3) fails then uniqueness might fail as well (see [AC]).

In this paper we would like to address the question of uniqueness for the approximation of (1.2). As we will see, this is not a trivial matter due, for instance, to the maximum principle. Indeed in order to prove uniqueness for problems such as (1.2) one needs to use more tricky test functions than the positive part of the difference of two solutions.

Let us denote by $V_{0}^{h}$ a finite-dimensional subspace of $H_{0}^{1}(\Omega)$. Then under the above assumptions let us introduce $u_{h}$, a solution to

$$
\left\{\begin{array}{l}
\int_{\Omega} a\left(x, u_{h}\right) \nabla u_{h} \cdot \nabla v d x=\langle f, v\rangle \quad \forall v \in V_{0}^{h}, \\
u_{h} \in V_{0}^{h},
\end{array}\right.
$$

where $\langle$,$\rangle denotes the duality bracket between H^{-1}(\Omega)$ and $H_{0}^{1}(\Omega)$. Then we have the following theorem.

\footnotetext{
${ }^{*}$ Received by the editors May 9, 1994; accepted for publication (in revised form) December 9, 1994.

†Centre d'Analyse Non Linéaire, Université de Metz, Ile du Saulcy, 57045 METZ Cedex 01, France.

$\ddagger$ Department of Mathematics, University Zurich-Irchel, Winterthurerstrasse 1908057 Zurich, Switzerland (chipot@amath.unizh.ch).
} 
THEOREM 1.2. If $f \in H^{-1}(\Omega)$ and if $a$ is a Carathéodory function satisfying (1.1), (1.3), then there exists a solution $u_{h}$ to (1.4).

Proof. For $v \in V_{0}^{h}$, due for instance to the Lax-Milgram theorem, there exists a unique $u$ solution to

$$
\left\{\begin{array}{l}
\int_{\Omega} a(x, v) \nabla u \cdot \nabla w d x=\langle f, w\rangle \quad \forall w \in V_{0}^{h} \\
u \in V_{0}^{h}
\end{array}\right.
$$

Let us assume $H_{0}^{1}(\Omega)$ and $V_{0}^{h}$ are equipped with the norm

$$
\|v\|^{2}=\int_{\Omega}|\nabla v(x)|^{2} d x=\|\nabla v\|_{2}^{2}
$$

where $|\cdot|$ denotes the euclidean norm and $|\cdot|_{2}$ the usual $L^{2}(\Omega)$-norm. Moreover, denote by $\|\cdot\|_{*}$ the dual norm in $H^{-1}(\Omega)$.

Taking $w=u$ in (1.5) we get, due to (1.1),

$$
\alpha \int_{\Omega}|\nabla u(x)|^{2} d x \leq \int_{\Omega} a(x, v) \nabla u \cdot \nabla u d x=\langle f, u\rangle \leq\|f\|_{*}\|u\| .
$$

Hence

$$
\|u\| \leq \frac{\|f\|_{*}}{\alpha}
$$

Then the map

$$
v \rightarrow T(v)=u
$$

maps the ball $B\left(0, \frac{\|f\|_{*}}{\alpha}\right)$ of $V_{0}^{h}$ into itself. Moreover, it is easy to show that this map is continuous. Thus, due to the Brouwer fixed-point theorem, the existence of a solution to (1.4) follows.

We would like to turn now to the question of uniqueness for a solution to (1.4). We will consider the case of $P_{1}$-Lagrange finite elements in dimension 1 and 2 , referring the reader to [A] for complements and other finite-element methods. Roughly speaking the results are the following: if $h$, the mesh size (see below), is small enough then there exists a unique solution to (1.4). If now $h$ is arbitrary then uniqueness to (1.4) might fail even if $a$ is Lipschitz continuous, i.e., even if the continuous problem (1.2) has a unique solution.

2. The one-dimensional case. In this section we assume that $\Omega=(a, b)$ and we consider a subdivision of $\Omega$

$$
a=a_{0}<a_{1}<\cdots<a_{n-1}<a_{n}=b .
$$

Moreover, we set

$$
h=\max _{i=1, \ldots, n} a_{i}-a_{i-1}
$$

We suppose $H_{0}^{1}(\Omega)$ is equipped with the norm

$$
\|u\|=\left|u^{\prime}\right|_{2}
$$

and $H^{-1}(\Omega)$ equipped with its dual norm $\|\cdot\|_{*}$. 
We set

$$
\begin{aligned}
& V_{0}^{h}=\{v: \Omega \rightarrow \mathbf{R}, v \text { is continuous on } \Omega, \\
& \left.\qquad v(a)=v(b)=0, v \text { is affine on each }\left(a_{i-1}, a_{i}\right)\right\} .
\end{aligned}
$$

It is clear that $V_{0}^{h}$ is a finite-dimensional subspace of $H_{0}^{1}(\Omega)$ of dimension $n-1$.

Then we have the following monotonicity property.

THEOREM 2.1. Assume that $f_{1}, f_{2} \in H^{-1}(\Omega)$ satisfy

$$
f_{1} \leq f_{2}
$$

in the $H^{-1}(\Omega)$ sense. Moreover assume that (1.1), (1.3) hold and that

$$
h^{\frac{1}{2}}<\frac{\alpha^{2}}{C\left\|f_{2}\right\|_{*}}
$$

where $C$ denotes the constant in (1.3). Then if $u_{i, h}, i=1,2$ denote solutions to

$$
\left\{\begin{array}{l}
\int_{\Omega} a\left(x, u_{i, h}\right) u_{i, h}^{\prime} v^{\prime} d x=\left\langle f_{i}, v\right\rangle \quad \forall v \in V_{0}^{h}, \\
u_{i, h} \in V_{0}^{h},
\end{array}\right.
$$

one has

$$
u_{1, h} \leq u_{2, h} .
$$

Proof. First notice that arguing as in (1.7), (1.8) one has for $i=1,2$

$$
\left\|u_{i, h}\right\|=\left|u_{i, h}^{\prime}\right|_{2} \leq \frac{\left\|f_{i}\right\|_{*}}{\alpha}
$$

Next if $u_{1, h}, u_{2, h}$ denote solutions to (2.7), by subtraction one gets

$$
\int_{\Omega} a\left(x, u_{1, h}\right) u_{1, h}^{\prime} v^{\prime} d x-\int_{\Omega} a\left(x, u_{2, h}\right) u_{2, h}^{\prime} v^{\prime} d x=\left\langle f_{1}-f_{2}, v\right\rangle \quad \forall v \in V_{0}^{h}
$$

Hence

$$
\begin{gathered}
\int_{\Omega} a\left(x, u_{1, h}\right)\left(u_{1, h}-u_{2, h}\right)^{\prime} v^{\prime} d x=\int_{\Omega}\left(a\left(x, u_{2, h}\right)-a\left(x, u_{1, h}\right)\right) u_{2, h}^{\prime} v^{\prime} d x \\
+\left\langle f_{1}-f_{2}, v\right\rangle \quad \forall v \in V_{0}^{h} .
\end{gathered}
$$

Setting

$$
w_{h}=u_{1, h}-u_{2, h}
$$

and using (1.3), we get

$$
\begin{aligned}
\int_{\Omega} a\left(x, u_{1, h}\right) w_{h}^{\prime} v^{\prime} d x & \leq \int_{\Omega}\left|a\left(x, u_{2, h}\right)-a\left(x, u_{1, h}\right)\left\|u_{2, h}^{\prime}\right\| v^{\prime}\right| d x+\left\langle f_{1}-f_{2}, v\right\rangle \\
& \leq \int_{\Omega} C\left|u_{2, h}-u_{1, h}\left\|u_{2, h}^{\prime}\right\| v^{\prime}\right| d x+\left\langle f_{1}-f_{2}, v\right\rangle \\
& \leq \int_{\Omega} C\left|w_{h}\left\|u_{2, h}^{\prime}\right\| v^{\prime}\right| d x+\left\langle f_{1}-f_{2}, v\right\rangle \quad \forall v \in V_{0}^{h} .
\end{aligned}
$$


Next we consider the function $v$ of $V_{0}^{h}$ defined by

$$
v\left(a_{i}\right)= \begin{cases}1 & \text { if } w_{h}\left(a_{i}\right)>0 \\ 0 & \text { else }\end{cases}
$$

Clearly, $v \geq 0$, so that by definition of the nonnegativity in the sense of $H^{-1}(\Omega)$ and (2.5) one has

$$
\left\langle f_{1}-f_{2}, v\right\rangle \leq 0
$$

and (2.13) reads

$$
\int_{\Omega} a\left(x, u_{1, h}\right) w_{h}^{\prime} v^{\prime} d x \leq C \int_{\Omega}\left|w_{h}\right|\left|u_{2, h}^{\prime} \| v^{\prime}\right| d x .
$$

Consider an interval $\left(a_{i-1}, a_{i}\right)$. On such an interval one has

$$
v^{\prime}=0
$$

unless

$$
w_{h}\left(a_{i-1}\right)>0 \text { and } w_{h}\left(a_{i}\right) \leq 0 \text { or } w_{h}\left(a_{i-1}\right) \leq 0 \text { and } w_{h}\left(a_{i}\right)>0 .
$$

Now if $\left(a_{i-1}, a_{i}\right)$ is an interval satisfying (2.18) one has, due to (2.14),

$$
w_{h}^{\prime} v^{\prime}=\frac{\left|w_{h}\left(a_{i}\right)-w_{h}\left(a_{i-1}\right)\right|}{\left(a_{i}-a_{i-1}\right)^{2}}>0 .
$$

So, from (1.1), (2.16), (2.17), (2.19) one deduces

$$
\alpha \sum_{i} \int_{a_{i-1}}^{a_{i}} \frac{\left|w_{h}\left(a_{i}\right)-w_{h}\left(a_{i-1}\right)\right|}{\left(a_{i}-a_{i-1}\right)^{2}} d x \leq C \sum_{i} \int_{a_{i-1}}^{a_{i}}\left|w_{h}\left(a_{i}\right)-w_{h}\left(a_{i-1}\right)\right|\left|u_{2, h}^{\prime} \| v^{\prime}\right| d x,
$$

where the summation is extended to the $i$ 's satisfying (2.18). We thus get

$$
\alpha \sum_{i} \frac{\left|w_{h}\left(a_{i}\right)-w_{h}\left(a_{i-1}\right)\right|}{a_{i}-a_{i-1}} \leq C \sum_{i} \frac{\left|w_{h}\left(a_{i}\right)-w_{h}\left(a_{i-1}\right)\right|}{a_{i}-a_{i-1}} \int_{a_{i-1}}^{a_{i}}\left|u_{2, h}^{\prime}\right| d x .
$$

By the Cauchy-Schwarz inequality and (2.9) one has

$$
\int_{a_{i-1}}^{a_{i}}\left|u_{2, h}^{\prime}\right| d x \leq\left|u_{2, h}^{\prime}\right|_{2}\left(a_{i}-a_{i-1}\right)^{\frac{1}{2}} \leq \frac{\left\|f_{2}\right\|_{*}}{\alpha} h^{\frac{1}{2}}
$$

and (2.21) becomes

$$
\alpha \sum_{i} \frac{\left|w_{h}\left(a_{i}\right)-w_{h}\left(a_{i-1}\right)\right|}{a_{i}-a_{i-1}} \leq \frac{C|| f_{i} \|_{*} h^{\frac{1}{2}}}{\alpha} \sum_{i} \frac{\left|w_{h}\left(a_{i}\right)-w_{h}\left(a_{i-1}\right)\right|}{a_{i}-a_{i-1}} .
$$

Thus if (2.6) holds, one obtains a contradiction unless the set of $i$ 's such that (2.18) holds is empty, i.e., unless

$$
w_{h}=u_{1, h}-u_{2, h} \leq 0 .
$$

This completes the proof of Theorem 2.1. 
As a consequence we can prove the next theorem.

THEOREM 2.2. Assume that $f \in H^{-1}(\Omega)$ and that a is a Carathéodory function satisfying (1.1), (1.3). Then if

$$
h^{\frac{1}{2}}<\frac{\alpha^{2}}{C\|f\|_{*}},
$$

where $C$ denotes the constant in (1.3), there exists a unique solution to (1.4). Moreover, if (2.24) fails then uniqueness can fail as well even if (1.3) holds, i.e., even if the continuous problem (1.2) has a unique solution.

Proof. Let $u_{1, h}, u_{2, h}$ be two solutions of (1.4). Applying Theorem 2.1 with $f_{1}=f_{2}=f$ we get

$$
u_{1, h}=u_{2, h}
$$

and uniqueness follows. To establish the second part of the theorem we are going to construct a function $a$ for which (1.4) will have two solutions.

Let us suppose for the sake of simplicity that $\Omega=(0,1)$ and

$$
a_{i}=i h \quad \forall i=0, \ldots, n \text {. }
$$

Then for $u \in V_{0}^{h}$ denote by

$$
u_{i}^{\prime}=\frac{u\left(a_{i}\right)-u\left(a_{i-1}\right)}{h} \quad \forall i=1, \ldots, n
$$

the slope of $u$ on $\left(a_{i-1}, a_{i}\right)$. Since

$$
0=\int_{0}^{1} u^{\prime} d x=\frac{1}{h} \sum_{1}^{n} u_{i}^{\prime}
$$

it is clear that every $u \in V_{0}^{h}$ is uniquely determined by $u_{i}^{\prime}$ 's such that

$$
\sum_{1}^{n} u_{i}^{\prime}=0
$$

Consider next the basis of $w_{i}$ of $V_{0}^{h}$ defined by

$$
w_{i}\left(a_{j}\right)=\delta_{i, j} \quad \forall i=1, \ldots, n-1, \quad \forall j=0, \ldots, n .
$$

Clearly (1.4) reads also

$$
\left\{\begin{array}{l}
\int_{\Omega} a\left(x, u_{h}\right) \nabla u_{h} \cdot \nabla w_{i} d x=\left\langle f, w_{i}\right\rangle \quad \forall i=1, \ldots, n-1, \\
u_{h} \in V_{0}^{h} .
\end{array}\right.
$$

Dropping for simplicity the subscript $h$, i.e., setting $u=u_{h}$ and

$$
f_{i}=\left\langle f, w_{i}\right\rangle, \quad \lambda_{i}^{u}=\frac{1}{h} \int_{a_{i-1}}^{a_{i}} a(x, u(x)) d x,
$$

it is clear that to solve (2.29) it is enough to find $u_{i}^{\prime}$ 's such that

$$
\left\{\begin{array}{l}
\lambda_{i}^{u} u_{i}^{\prime}-\lambda_{i+1}^{u} u_{i+1}^{\prime}=f_{i} \quad \forall i=1, \ldots, n-1, \\
\sum_{1}^{n} u_{i}^{\prime}=0
\end{array}\right.
$$


where if we set

$$
\begin{gathered}
u_{i}=u\left(a_{i}\right), \\
\lambda_{i}^{u}=\int_{0}^{1} a\left(h(t+i-1), u_{i-1}+t h u_{i}^{\prime}\right) d t .
\end{gathered}
$$

Consider next a smooth function $\varphi \in C^{\infty}(0,1)$ such that

$$
\left\{\begin{array}{l}
\varphi(0)=\varphi(1)=1, \\
\varphi^{\prime}(0)=\varphi^{\prime}(1)=0, \\
\int_{0}^{1} \varphi(t) d t=\frac{1}{2}, \\
\frac{1}{3} \leq \varphi(t) \leq 1 \quad \forall t \in(0,1) .
\end{array}\right.
$$

Clearly such a function does exist. Let $u \in V_{0}^{h}$ be defined by $u_{0}=u_{1}=0$ and

$$
u\left(a_{i}\right)=u_{i}>0 .
$$

Let us then construct $a$ from $u$. More precisely, for $x \in\left[a_{i-1}, a_{i}\right]$ set $t=\left(x-a_{i-1}\right) / h$ and

$$
a(x, z)=\left\{\begin{array}{l}
1 \text { for } z \leq u(x) \\
\varphi(t) \text { for } z \geq 2 u(x) \\
\mu+(1-\mu) \varphi(t) \text { for } z=\mu u(x)+2(1-\mu) u(x) \in[u(x), 2 u(x)] .
\end{array}\right.
$$

It is clear that $a \in\left[\frac{1}{3}, 1\right]$ so that (1.1) holds. Moreover, since $\varphi(0)=\varphi(1)=1$ and $u$ is continuous, $a$ is continuous. $a$ is also Lipschitz continuous in $z$. To see it, it is enough to notice that for $x \in\left[a_{i-1}, a_{i}\right]$ one has for almost every $z$

$$
\frac{\partial a}{\partial z}(x, z)=0 \quad \text { or } \quad \frac{\partial a}{\partial z}(x, z)=\frac{\varphi(t)-1}{u(x)} .
$$

In this later case for $i=1,2, \ldots, n-1$

$$
\left|\frac{\partial a}{\partial z}(x, z)\right| \leq \frac{1}{u(x)} \leq \frac{1}{\inf _{i} u_{i}} .
$$

Now, on $\left[a_{0}, a_{1}\right]$,

$$
\left|\frac{\partial a}{\partial z}(x, z)\right|=\left|\frac{\varphi(t)-1}{t u_{1}}\right|=\left|\frac{\varphi(t)-\varphi(0)}{t u_{1}}\right| \leq \frac{\max _{[0,1]}\left|\varphi^{\prime}(t)\right|}{u_{1}} .
$$

By a similar argument holding on $\left[a_{n-1}, a_{n}\right]$, it is clear from (2.37), (2.38) that (1.3) holds. It is also possible to show that $a$ is Lipschitz continuous in $x$ (see [A]).

Now, clearly

$$
f=-\left(a(x, u(x)) u^{\prime}\right)^{\prime} \in H^{-1}(\Omega) .
$$

In fact one can even find a piecewise constant function $g$ such that

$$
f_{i}=\left\langle f, w_{i}\right\rangle=\left\langle g, w_{i}\right\rangle \quad \forall i=1, \ldots, n-1 .
$$

Then $u$ and $v=2 u$ are both solutions to (2.31) with $f_{i}$ given by (2.40). Indeed

$$
\lambda_{i}^{u}=\frac{1}{h} \int_{a_{i-1}}^{a_{i}} a(x, u(x)) d x=1
$$


and

$$
\begin{aligned}
\lambda_{i}^{v} & =\frac{1}{h} \int_{a_{i-1}}^{a_{i}} a(x, v(x)) d x \\
& =\int_{0}^{1} a(h(t+(i-1)), 2 u(h(t+(i-1)))) d t \\
& =\int_{0}^{1} \varphi(t) d t=\frac{1}{2} .
\end{aligned}
$$

Thus since $v^{\prime}=2 u^{\prime}$,

$$
\lambda_{i}^{u} u_{i}^{\prime}-\lambda_{i+1}^{u} u_{i+1}^{\prime}=\lambda_{i}^{v} v_{i}^{\prime}-\lambda_{i+1}^{v} v_{i+1}^{\prime} \quad \forall i=1, \ldots, n-1,
$$

and the result follows.

Remark 2.1. Note that in the proof of uniqueness we did not assume the mesh to be uniform, i.e., we did not assume that

$$
a_{i}-a_{i-1} \geq c \cdot h
$$

for some positive constant $c$.

3. A convergence result. In this section we assume that $\Omega$ is a bounded subset of $\mathbf{R}^{n}$, $n \geq 1$. We denote by $V_{0}^{h}, h>0$ a subspace of $H_{0}^{1}(\Omega)$ of finite dimension and we consider $u_{h}$ a solution to

$$
\left\{\begin{array}{l}
\int_{\Omega} a\left(x, u_{h}\right) \nabla u_{h} \cdot \nabla v d x=\langle f, v\rangle \quad \forall v \in V_{0}^{h}, \\
u_{h} \in V_{0}^{h} .
\end{array}\right.
$$

Moreover, we assume that

$$
\forall v \in H_{0}^{1}(\Omega), \exists v_{h} \in V_{0}^{h} \quad \text { such that } \quad v_{h} \rightarrow v \text { in } H_{0}^{1}(\Omega) \text { when } h \rightarrow 0 .
$$

Then we have the following theorem.

THEOREM 3.1. Assume that $f \in H^{-1}(\Omega)$ and that a is a Carathéodory function satisfying (1.1), (1.3). Then if $u_{h}$ is a solution to (3.1) and if (3.2) holds one has

$$
\lim _{h \rightarrow 0} u_{h}=u
$$

in $H_{0}^{1}(\Omega)$-strong. $u$ denotes the unique weak solution to (1.2).

Proof. First, arguing as in (1.8), we get

$$
\left\|u_{h}\right\| \leq \frac{\|f\|_{*}}{\alpha}
$$

so that, up to a subsequence, one has

$$
\begin{gathered}
u_{h} \rightarrow u_{0} \text { in } H_{0}^{1}(\Omega), \\
u_{h} \rightarrow u_{0} \text { in } L^{2}(\Omega)
\end{gathered}
$$

when $h \rightarrow 0$. (Recall that $H_{0}^{1}(\Omega)$ is compactly imbedded in $L^{2}(\Omega)$.) So, again up to a subsequence, one has

$$
u_{h} \rightarrow u_{0} \text { a.e. in } \Omega
$$


and

$$
a\left(x, u_{h}\right) \rightarrow a\left(x, u_{0}\right) \text { a.e. in } \Omega .
$$

Let $v \in H_{0}^{1}(\Omega)$. By (3.2) there exists a "sequence" $v_{h} \in V_{0}^{h}$ such that

$$
\nabla v_{h} \rightarrow \nabla v \text { in }\left(L^{2}(\Omega)\right)^{n} .
$$

Then one has

$$
a\left(x, u_{h}\right) \nabla v_{h} \rightarrow a\left(x, u_{0}\right) \nabla v \text { in }\left(L^{2}(\Omega)\right)^{n} .
$$

Indeed, this follows from the inequality

$$
\begin{aligned}
\int_{\Omega}\left|a\left(x, u_{h}\right) \nabla v_{h}-a\left(x, u_{0}\right) \nabla v\right|^{2} d x \leq & 2 \int_{\Omega}\left|a\left(x, u_{h}\right)\left(\nabla v_{h}-\nabla v\right)\right|^{2} d x \\
& +2 \int_{\Omega}\left|a\left(x, u_{h}\right)-a\left(x, u_{0}\right)\right|^{2}|\nabla v|^{2} d x \\
\leq & 2 \beta^{2} \int_{\Omega}\left|\left(\nabla v_{h}-\nabla v\right)\right|^{2} d x \\
& +2 \int_{\Omega}\left|a\left(x, u_{h}\right)-a\left(x, u_{0}\right)\right|^{2}|\nabla v|^{2} d x
\end{aligned}
$$

by the Lebesgue convergence theorem (see (3.8), (3.9)). From (3.1), i.e., from

$$
\int_{\Omega} a\left(x, u_{h}\right) \nabla u_{h} \cdot \nabla v_{h} d x=\left\langle f, v_{h}\right\rangle
$$

and by (3.10), (3.5), one has passing to the limit

$$
\int_{\Omega} a\left(x, u_{0}\right) \nabla u_{0} \cdot \nabla v d x=\langle f, v\rangle \quad \forall v \in H_{0}^{1}(\Omega) .
$$

Thus $u_{0}=u$, the unique weak solution to (1.2). Since we have only one possible limit for any subsequence of $u_{h}$, we have

$$
\begin{aligned}
& u_{h} \rightarrow u \text { in } H_{0}^{1}(\Omega), \\
& u_{h} \rightarrow u \text { in } L^{2}(\Omega) .
\end{aligned}
$$

We then derive by $(1.1)$

$$
\begin{aligned}
\alpha \int_{\Omega}\left|\nabla u_{h}-\nabla u\right|^{2} d x & \leq \int_{\Omega} a\left(x, u_{h}\right)\left|\nabla u_{h}-\nabla u\right|^{2} d x \\
& =\int_{\Omega} a\left(x, u_{h}\right)\left|\nabla u_{h}\right|^{2} d x-2 \int_{\Omega} a\left(x, u_{h}\right) \nabla u_{h} \cdot \nabla u d x \\
& +\int_{\Omega} a\left(x, u_{h}\right)|\nabla u|^{2} d x
\end{aligned}
$$

By (3.1)

$$
\int_{\Omega} a\left(x, u_{h}\right) \nabla u_{h} \cdot \nabla u_{h} d x=\left\langle f, u_{h}\right\rangle
$$


so that

$$
\lim _{h \rightarrow 0} \int_{\Omega} a\left(x, u_{h}\right) \nabla u_{h} \cdot \nabla u_{h} d x=\langle f, u\rangle=\int_{\Omega} a(x, u) \nabla u \cdot \nabla u d x .
$$

Next, the only possible limits for

$$
\int_{\Omega} a\left(x, u_{h}\right) \nabla u_{h} \cdot \nabla u d x \quad \text { and } \quad \int_{\Omega} a\left(x, u_{h}\right)|\nabla u|^{2} d x
$$

are for both

$$
\int_{\Omega} a(x, u)|\nabla u|^{2} d x
$$

Indeed, if the above integrals converge, up to a subsequence one has

$$
u_{h} \rightarrow u \text { a.e. in } \Omega \text {. }
$$

Thus by the Lebesgue theorem

$$
a\left(x, u_{h}\right) \nabla u \rightarrow a(x, u) \nabla u \text { in }\left(L^{2}(\Omega)\right)^{n} .
$$

Using (3.13) we can then pass to the limit in (3.17) to get for both limits (3.18). Since (3.17) has only one possible limit one obtains

$$
\lim _{h \rightarrow 0} \int_{\Omega} a\left(x, u_{h}\right) \nabla u_{h} \cdot \nabla u d x=\lim _{h \rightarrow 0} \int_{\Omega} a\left(x, u_{h}\right)|\nabla u|^{2} d x=\int_{\Omega} a(x, u)|\nabla u|^{2} d x .
$$

Recalling (3.15), (3.16), (3.20) one gets

$$
\lim _{h \rightarrow 0} \int_{\Omega}\left|\nabla u_{h}-\nabla u\right|^{2} d x=0
$$

and the result follows.

4. A two-dimensional case. In this section we assume that $\Omega$ is a polygonal domain of $\mathbf{R}^{2}$ with boundary $\Gamma$. We denote by $\tau_{h}$ a regular triangulation of $\Omega$ (see for instance [C]). Recall that

$$
h=\max _{K \in \tau_{h}} h_{K},
$$

where $h_{K}$ denotes the diameter of the triangle $K$. In fact we will need a slightly stronger assumption than for $\tau_{h}$ to be regular. More precisely, we will not allow triangles with angles larger then $\frac{\pi}{2}$, i.e., if $\theta_{i}^{K}, i=1,2,3$ denote the angles of the triangle $K$ we will assume that for some constant $\delta$ one has

$$
0<\delta<\theta_{i}^{K} \leq \frac{\pi}{2}-\delta \quad \forall K \in \tau_{h} \quad \forall i=1,2,3 .
$$

Then denote by $V_{0}^{h}$ the finite-dimensional subspace of $H_{0}^{1}(\Omega)$ defined by

$$
V_{0}^{h}=\left\{v: \Omega \rightarrow \mathbf{R} \text {, continuous, } v=0 \text { on } \Gamma,\left.v\right|_{K} \in P_{1} \forall K \in \tau_{h}\right\},
$$

where $P_{1}$ denotes the space of polynomials of degree $1,\left.v\right|_{K}$ the restriction of $v$ to $K$.

Then we have the following theorem. 
THEOREM 4.1. Assume that $f \in H^{-1}(\Omega)$, a is a Carathéodory function satisfying (1.1), (1.3) and that (4.2) holds. Then if $h$ is small enough the approximated problem (1.4) has a unique solution. In the case where $h$ is arbitrary, then uniqueness might fail even if the continuous problem (1.2) has a unique solution.

Before giving the proof of Theorem 4.1, we will need a lemma.

For $K \in \tau_{h}$ denote by $K_{1}, K_{2}, K_{3} \in \mathbf{R}^{2}$ the vertices of $K$ and by $\lambda_{K_{i}}$ the affine functions such that

$$
\lambda_{K_{i}}\left(K_{j}\right)=\delta_{i, j} \quad \forall i, j=1,2,3 .
$$

Then we can prove the next lemma.

LEMMA 4.1. Assume that $K$ is a triangle that satisfies (4.2). Then, for any $i, j=1,2,3$ there exist constants $c_{1}, c_{2}>0$ such that

$$
\begin{gathered}
\nabla \lambda_{K_{i}} \cdot \nabla \lambda_{K_{j}} \leq-\frac{c_{1}}{h_{K}^{2}}<0 \quad \forall i \neq j, \\
\frac{1}{h_{K}} \leq\left|\nabla \lambda_{K_{i}}\right| \leq \frac{c_{2}}{h_{K}} .
\end{gathered}
$$

Proof. Without loss of generality we can assume that $i=2, j=3, K_{1}=0$. Denote by $R$ the rotation of angle $\frac{\pi}{2}$ and by (.) the scalar product in $\mathbf{R}^{2}$. One has clearly

$$
\lambda_{K_{2}}(x)=\left(x \cdot R\left(K_{3}\right)\right) /\left(K_{2} \cdot R\left(K_{3}\right)\right), \quad \lambda_{K_{3}}(x)=\left(x \cdot R\left(K_{2}\right)\right) /\left(K_{3} \cdot R\left(K_{2}\right)\right)
$$

so that

$$
\nabla \lambda_{K_{2}} \cdot \nabla \lambda_{K_{3}}=\left(R\left(K_{2}\right) \cdot R\left(K_{3}\right)\right) /\left(K_{2} \cdot R\left(K_{3}\right)\right)\left(K_{3} \cdot R\left(K_{2}\right)\right) .
$$

If $\theta_{1}$ denotes the angle of $K$ at $K_{1}$ one has

$$
\begin{gathered}
\left(R\left(K_{2}\right) \cdot R\left(K_{3}\right)\right)=\left(K_{2} \cdot K_{3}\right)=\cos \theta_{1}\left|K_{2}\right|\left|K_{3}\right|>0, \\
\left(K_{2} \cdot R\left(K_{3}\right)\right)=-\sin \theta_{1}\left|K_{2}\right|\left|K_{3}\right|<0, \\
\left(K_{3} \cdot R\left(K_{2}\right)\right)=\sin \theta_{1}\left|K_{2}\right|\left|K_{3}\right|>0 .
\end{gathered}
$$

It follows that

$$
\nabla \lambda_{K_{2}} \cdot \nabla \lambda_{K_{3}}=-\frac{\cos \theta_{1}}{\sin ^{2} \theta_{1}\left|K_{2}\right|\left|K_{3}\right|} .
$$

Then, (4.5) follows from (4.2).

To get (4.6) note that

$$
\left|\nabla \lambda_{K_{2}}\right|=\frac{\left|R\left(K_{3}\right)\right|}{\left|\left(K_{2} \cdot R\left(K_{3}\right)\right)\right|}=\frac{\left|K_{3}\right|}{\sin \theta_{1}\left|K_{2}\right|\left|K_{3}\right|}=\frac{1}{\sin \theta_{1}\left|K_{2}\right|} .
$$

Due to (4.2), the result follows. Let us now turn to the proof of Theorem 4.1.

Proof of Theorem 4.1. Let $u_{i, h}, i=1,2$ be two solutions to (1.4). By subtraction one gets

$$
\begin{aligned}
\int_{\Omega} a\left(x, u_{1, h}\right) \nabla & \left(u_{1, h}-u_{2, h}\right) \cdot \nabla v d x \\
= & \int_{\Omega}\left(a\left(x, u_{2, h}\right)-a\left(x, u_{1, h}\right)\right) \nabla u_{2, h} \nabla v d x \quad \forall v \in V_{0}^{h} .
\end{aligned}
$$


Setting

$$
w_{h}=u_{1, h}-u_{2, h}
$$

and using (1.3) we obtain

$$
\int_{\Omega} a\left(x, u_{1, h}\right) \nabla w_{h} \cdot \nabla v d x \leq C \int_{\Omega}\left|w_{h}\right|\left|\nabla u_{2, h}\right||\nabla v| d x \quad \forall v \in V_{0}^{h} .
$$

Consider next the function $v$ of $V_{0}^{h}$ defined by

$$
v\left(K_{i}\right)= \begin{cases}1 & \text { if } w_{h}\left(K_{i}\right)>0 \\ 0 & \text { else }\end{cases}
$$

On a triangle $K$ one has

$$
\nabla v=0
$$

unless $K$ is such that

$$
w_{h}\left(K_{i}\right)>0, \quad w_{h}\left(K_{j}\right) \leq 0, \quad w_{h}\left(K_{k}\right) \leq 0
$$

or

$$
w_{h}\left(K_{i}\right)>0, \quad w_{h}\left(K_{j}\right)>0, \quad w_{h}\left(K_{k}\right) \leq 0 .
$$

In the triplet $(i, j, k)$ the entries must be different and take the value $1,2,3$.

So from (4.12) we deduce

$$
\sum_{K} \int_{K} a\left(x, u_{1, h}\right) \nabla w_{h} \cdot \nabla v d x \leq C \sum_{K} \int_{K}\left|w_{h}\right|\left|\nabla u_{2, h} \| \nabla v\right| d x,
$$

where the summation is extended to the $K$ 's satisfying (4.14), (4.15). Now if $K$ satisfies (4.14) or (4.15) it is clear that $w_{h}$ vanishes in $K$ at some point $y$ and one has

$$
w_{h}(x)=w_{h}(x)-w_{h}(y)=\nabla w_{h} \cdot(x-y) \quad \forall x \in K
$$

and thus

$$
\left|w_{h}(x)\right| \leq\left|\nabla w_{h}\right| h_{K} \forall x \in K
$$

Recalling (4.16) we obtain

$$
\sum_{K} \int_{K} a\left(x, u_{1, h}\right) \nabla w_{h} \cdot \nabla v d x \leq C \sum_{K} h_{K} \int_{K}\left|\nabla w_{h}\right|\left|\nabla u_{2, h}\right||\nabla v| d x
$$

Let us set $a_{i}^{K}=w_{h}\left(K_{i}\right)$ so that on each $K \in \tau_{h}$

$$
w_{h}=a_{1}^{K} \lambda_{K_{1}}+a_{2}^{K} \lambda_{K_{2}}+a_{3}^{K} \lambda_{K_{3}} .
$$

Let us assume that $K$ is a triangle such that (4.14) holds. Let us assume for instance that

$$
a_{1}^{K}>0, \quad a_{2}^{K} \leq 0, \quad a_{3}^{K} \leq 0 .
$$


Then on $K$ one has $v=\lambda_{K_{1}}$ and thus by (4.6), (4.7), (4.19)

$$
\begin{aligned}
\nabla w_{h} \cdot \nabla v & =a_{1}^{K}\left|\nabla \lambda_{K_{1}}\right|^{2}+a_{2}^{K} \nabla \lambda_{K_{2}} \cdot \nabla \lambda_{K_{1}}+a_{3}^{K} \nabla \lambda_{K_{3}} \cdot \nabla \lambda_{K_{1}} \\
& \geq \frac{c}{h_{K}^{2}}\left(\left|a_{1}^{K}\right|+\left|a_{2}^{K}\right|+\left|a_{3}^{K}\right|\right),
\end{aligned}
$$

where $c=\min \left(c_{1}, 1\right)$ is independent of $K$.

If now we are in a case where (4.15) holds, i.e., if for instance

$$
a_{1}^{K}>0, \quad a_{2}^{K}>0, \quad a_{3}^{K} \leq 0,
$$

then

$$
v=\lambda_{K_{1}}+\lambda_{K_{2}}=1-\lambda_{K_{3}}
$$

and

$$
\begin{aligned}
\nabla w_{h} \cdot \nabla v & =-a_{1}^{K} \nabla \lambda_{K_{1}} \cdot \nabla \lambda_{K_{3}}-a_{2}^{K} \nabla \lambda_{K_{2}} \cdot \nabla \lambda_{K_{3}}-a_{3}^{K}\left|\nabla \lambda_{K_{3}}\right|^{2} \\
& \geq \frac{c}{h_{K}^{2}}\left(\left|a_{1}^{K}\right|+\left|a_{2}^{K}\right|+\left|a_{3}^{K}\right|\right),
\end{aligned}
$$

where, as above, $c=\min \left(c_{1}, 1\right)$ is independent of $K$. Now by (4.18), (4.6) one has on each triangle $K$

$$
\left|\nabla w_{h}\right| \leq \frac{c_{2}}{h_{K}}\left(\left|a_{1}^{K}\right|+\left|a_{2}^{K}\right|+\left|a_{3}^{K}\right|\right), \quad|\nabla v| \leq \frac{c_{2}}{h_{K}} .
$$

Recalling (1.3), (4.17), and (4.19)-(4.22) we obtain

$$
\alpha c \sum_{K} \frac{1}{h_{K}^{2}} \int_{K}\left|a_{1}^{K}\right|+\left|a_{2}^{K}\right|+\left|a_{3}^{K}\right| d x \leq C c_{2}^{2} \sum_{K} \frac{1}{h_{K}} \int_{K}\left(\left|a_{1}^{K}\right|+\left|a_{2}^{K}\right|+\left|a_{3}^{K}\right|\right)\left|\nabla u_{2, h}\right| d x .
$$

Hence, for some constant $M$,

$$
\sum_{K}\left|a_{1}^{K}\right|+\left|a_{2}^{K}\right|+\left|a_{3}^{K}\right| \leq M \sum_{K} \frac{\left|a_{1}^{K}\right|+\left|a_{2}^{K}\right|+\left|a_{3}^{K}\right|}{h_{K}} \int_{K}\left|\nabla u_{2, h}\right| d x .
$$

Using the Cauchy-Schwarz inequality it follows that

$$
\sum_{K}\left|a_{1}^{K}\right|+\left|a_{2}^{K}\right|+\left|a_{3}^{K}\right| \leq M \sum_{K}\left(\left|a_{1}^{K}\right|+\left|a_{2}^{K}\right|+\left|a_{3}^{K}\right|\right)|| \nabla u_{2, h}||_{2, K},
$$

where $|\cdot|_{p, A}$ denotes the usual $L^{p}(A)$-norm. If $u$ denotes the solution to (1.2) then

$$
\begin{aligned}
\left\|\nabla u_{2, h}\right\|_{2, K} & \leq\left\|\nabla\left(u_{2, h}-u\right)\right\|_{2, K}+\|\nabla u\|_{2, K} \\
& \leq\left\|\nabla\left(u_{2, h}-u\right)\right\|_{2}+\|\nabla u\|_{2, K}=\epsilon(h),
\end{aligned}
$$

where $\epsilon(h) \rightarrow 0$ when $h \rightarrow 0$ (see Theorem 3.1). So (4.24) reads

$$
\sum_{K}\left|a_{1}^{K}\right|+\left|a_{2}^{K}\right|+\left|a_{3}^{K}\right| \leq \epsilon(h) M \sum_{K}\left|a_{1}^{K}\right|+\left|a_{2}^{K}\right|+\left|a_{3}^{K}\right|
$$

and is a contradiction when $h$ is small enough unless the set of $K$ satisfying (4.14), (4.15) is empty, i.e., unless $w_{h} \leq 0$. Exchanging the roles of $u_{1, h}$ and $u_{2, h}$, the uniqueness result follows. 
Remark 4.1. We did not assume here that the mesh be uniform (see (2.41)). Arguing as in Theorem 2.1 we could have gotten a monotonicity result in terms of $f$.

We now turn to the construction of an example for which uniqueness fails. The proof in essence is identical to the one in dimension $1 . \Omega$ will be an equilateral triangle in $\mathbf{R}^{2}$ of side 1 and $\tau_{h}$ a triangulation of $\Omega$ made of equilateral triangles of same sides $h . V_{0}^{h}$ is the space defined in (4.3). Then a solution $u_{h}$ to (1.4) is such that

$$
\int_{\Omega} a\left(x, u_{h}\right) \nabla u_{h} \cdot \nabla w d x=\sum_{K \in \tau_{h}} \nabla u_{h} \cdot \nabla w \int_{K} a\left(x, u_{h}\right) d x \quad \forall w \in V_{0}^{h},
$$

since $\nabla u_{h}, \nabla w$ are constant on each $K \in \tau_{h}$.

Let us define a function $\varphi$ on $\Omega$ that is $C^{\infty}$, invariant by the isometries of $\Omega$, and that satisfies

$$
\left\{\begin{array}{l}
\varphi=1 \text { on } \Gamma \\
|\nabla \varphi|=0 \text { on } \Gamma \\
\int_{\Omega} \varphi=\frac{\sqrt{3}}{8} \\
\frac{1}{4} \leq \varphi \leq 1
\end{array}\right.
$$

Let $u \in V_{0}^{h}$ be such that

$$
u>0 \text { at the nodes inside } \Omega \text {. }
$$

For every $K \in \tau_{h}$ denote by $y_{K}$ an affine one-to-one map that assigns to every point $x \in K$ the point $y_{K}(x) \in \Omega$ having the same barycentric coordinates as $x$.

From $u$ one constructs $a$ by setting on each triangle $K$

$$
a(x, z)=\left\{\begin{array}{l}
1 \text { for } z \leq u(x), \\
\varphi\left(y_{K}(x)\right) \text { for } z \geq 2 u(x), \\
\mu+(1-\mu) \varphi\left(\left(y_{K}(x)\right) \text { for } z=\mu u(x)+2(1-\mu) u(x) \in[u(x), 2 u(x)] .\right.
\end{array}\right.
$$

It is clear then that

$$
\begin{gathered}
\int_{K} a(x, u(x)) d x=\frac{\sqrt{3}}{4} h^{2} \quad \forall K \in \tau_{h}, \\
\int_{K} a(x, v(x)) d x=\int_{K} a(x, 2 u(x)) d x=\int_{K} \varphi\left(y_{K}(x)\right) d x \\
=\int_{K} \varphi\left(y_{K}(x)\right) d x=\frac{\sqrt{3}}{8} h^{2} \quad \forall K \in \tau_{h} .
\end{gathered}
$$

Thus (see (4.26)), it is clear that $u, v$ are such that

$$
\int_{\Omega} a(x, u) \nabla u \cdot \nabla w d x=\int_{\Omega} a(x, v) \nabla v \cdot \nabla w d x \quad \forall w \in V_{0}^{h}
$$

Now, clearly

$$
w \rightarrow \int_{\Omega} a(x, u) \nabla u \cdot \nabla w d x
$$

is in $H^{-1}(\Omega)$ so that $u, v$ are both a solution to (1.4) for $f$ given by (4.32). 
It remains to show that $a$ is Lipschitz continuous in $z$. The arguments are the same as in the one-dimensional case and we only sketch them. First note that on each $K \in \tau_{h}$

$$
\frac{\partial a}{\partial z}(x, z)=0 \quad \text { or } \quad \frac{\partial a}{\partial z}(x, z)=\frac{\varphi\left(y_{K}(x)\right)-1}{u(x)} .
$$

So if $K \in \tau_{h}$ does not touch the boundary of $\Omega$, by (4.28)

$$
\left|\frac{\partial a}{\partial z}(x, z)\right| \leq \frac{1}{u(x)} \leq C .
$$

If now $K \in \tau_{h}$ is touching the boundary of $\Omega$ and not at the corners, since $u$ is affine, one has for some positive constant $m$

$$
u(x) \geq m \operatorname{dist}(x, \Gamma)=m h \operatorname{dist}\left(y_{K}(x), \Gamma\right) .
$$

Moreover, by the mean value theorem

$$
\left|\varphi\left(y_{K}(x)\right)-1\right|=\left|\varphi\left(y_{K}(x)\right)-\varphi(0)\right| \leq C \operatorname{dist}\left(y_{K}(x), \Gamma\right) .
$$

It follows from (4.34) that

$$
\left|\frac{\partial a}{\partial z}(x, z)\right| \leq \frac{C}{m h}
$$

which completes the proof.

In this note we have only addressed cases where the results are complete and simple. We refer to forthcoming papers and $[\mathrm{A}]$ for more cases.

\section{REFERENCES}

[A] N. ANDRÉ, Sur l'unicité de problèmes quasilinéaires elliptiques et de leur approximations numériques, Ph.D. thesis, University of Metz, Ile du Saulcy, France, 1993.

[AC] N. ANDRÉ AND M. CHIPOT, A remark on uniqueness for quasilinear elliptic equations, in Proc. of the Banach Center, to appear.

[Ar] M. ARTOLA, Sur une classe de problèmes paraboliques quasilinéaires, Boll. Un. Mat. Ital. B, 6 (1986), pp. $51-70$.

[BKS] H. BRezis, D. Kinderlehrer, AND G. Stampacchia, Sur une nouvelle formulation du problème de l'écoulement à travers une digue, C. R. Acad. Sci. Paris Sér. A, 287 (1978), pp. 711-714.

[C] P. G. CIARLET, The Finite Element Method for Elliptic Problems, North-Holland, Amsterdam, 1987.

[CC] J. CARRILlo AND M. CHIPOT, On nonlinear elliptic equations involving derivative of the nonlinearity, in Proc. Roy. Soc. Edinburgh Sect., A 100 (1985), pp. 281-294.

[CM] M. CHIPOT AND G. MichaIlLe, Uniqueness results and monotonicity properties for the solution of some variational inequalities, Ann. Scuola Norm. Sup. Pisa Cl. Sci. (4), 16 (1989), pp. 137-166.

[GT] D. Gilbarg And N. S. TRudinger, Elliptic Partial Differential Equations of Second Order, Springer-Verlag, Berlin, New York, 1985.

[KS] D. Kinderlehrer and G. Stampacchia, An Introduction to Variational Inequalities, Academic Press, New York, 1980.

[M] G. MichaILle, Inéquations variationnelles fortement non linéaires et applications, Ph.D. thesis, University of Metz, Ile du Saulcy, France, 1988.

[T] N. S. TRUdinger, On the comparison principle for quasilinear divergence structure equations, Arch. Rational Mech. Anal., 57 (1974), pp. 128-133. 